\title{
Interference Alignment with Combined Receivers For Heterogeneous Networks
}

\author{
Refik Fatih Ustok*, Mansoor Shafi ${ }^{\ddagger}$, Pawel A. Dmochowski* and Peter J. Smith ${ }^{\dagger}$ \\ ${ }^{*}$ School of Engineering and Computer Science, Victoria University of Wellington, Wellington, New Zealand \\ ${ }^{\dagger}$ Department of Electrical and Computer Engineering, University of Canterbury, Christchurch, New Zealand \\ $\ddagger$ Telecom New Zealand, Wellington, New Zealand \\ Emails: \{refik.ustok, pawel.dmochowski\}@ecs.vuw.ac.nz, mansoor.shafi@telecom.co.nz, peter.smith@canterbury.ac.nz
}

\begin{abstract}
We propose a novel combined receiver which applies zero forcing $(\mathrm{ZF})$ to null the dominant interferers, followed by minimum-mean-square-error (MMSE) processing to reduce the effect of the remaining interference and noise. The receiver is used in conjunction with an interference alignment (IA) scheme in a multicell environment. Simulation results considering a range of interferer profiles show that the combined receiver outperforms an MMSE receiver, due to its ability to eliminate the dominant interferers. The complete nulling of many dominant interferers in a multi-user, multicell case requires a large number of receive antennas. If the antennas are closely located in a compact array, then spatial correlation is important and its impact on the IA scheme is also considered.
\end{abstract}

\section{INTRODUCTION}

Future mobile wireless networks are faced with an increasing demand for higher data rates. Recent research shows that the mobile data volume will increase by a factor of 18 in the next five years [1]. Hence, more efficient communication systems must be developed where multiple transmitter and receiver pairs use the same radio resources. This resource sharing inherently leads to increased interference. The traditional approaches to mitigating interference focus on partitioning the total radio resources, and are inherently limited in the number of users they can support.

Recently, researchers have proposed a new technique [2], [3] called Interference Alignment (IA). With IA, under certain conditions, each user can utilize one half of the network resources interference free, regardless of how many users exist in the network. IA represents a breakthrough as it provides more capacity than was previously thought possible. The key idea of IA is to fit the undesired signals from various users into a smaller signal space and separate it from the desired signals [4].

IA has been studied in relay aided networks [5], [10] and also for cellular networks. In [6], a two cell interfering twouser Multiple-Input-Multiple-Output (MIMO) uplink system with limited feedback is considered. The main contribution of [7] is a subspace interference alignment scheme for a two cell downlink system. Here, interference is aligned into a multidimensional subspace. The proposed scheme in [7] requires the number of dimensions to grow linearly with the user numbers in each cell. In [8], the authors developed an IA technique for a downlink-cellular system using a receiver that mimics MMSE. The scheme provides a substantial gain as compared to matched filtering and $\mathrm{ZF}$, especially when there are dominant interferers and other non-negligible interferers.

It is well known that MMSE receivers do not null the dominant interferers completely. $\mathrm{ZF}$ receivers on the other hand can remove some interferers completely, but require sufficient signal dimensions to perform this nulling. In this paper, we propose combining the function of both ZF and MMSE receivers. The $\mathrm{ZF}$ part nulls out the dominant interferers completely while the MMSE part reduces the remaining, nonnegligible interferers and noise. This is motivated by the fact that a receiver in a multi-user scenario typically experiences a small number of dominant interferers.

In this paper, we consider a heterogenous uplink network with a macro-pico cell deployment. A macrocell can be considered as a large area cell where radio coverage is served by a high power cellular base station (BS). A picocell is served by a small, low-power cellular base station, covering a smaller area, such as an in-building location or highly populated areas. In cellular networks, picocells are typically used to extend coverage to indoor areas with low signal strength, or to add network capacity in areas with very dense usage, such as train stations, shopping malls, etc. When users are connected to both macrocell $\mathrm{BS}$ and picocell $\mathrm{BS}$ then the picocell $\mathrm{BS}$ can see significant interference from users located in nearby macrocells and also in neighbouring picocells. This interference can be much stronger than the aggregate interference from the remaining macrocells and picocells, especially when the distance between the interferer and the victim receiver is small. The idea of the combined receivers is to null out the dominant interferers via $\mathrm{ZF}$, and subsequently reduce the remaining interference by the MMSE technique.

Our contributions in this paper are as follows:

- We consider an IA scheme with a novel combined receiver which nulls the dominant interferers with $\mathrm{ZF}$ and subsequently uses MMSE processing to reduce the effects of the remaining interference and noise.

- We implement our scheme in an IA heterogeneous uplink network which involves a mix of macro and pico cells.

- We show the range of remaining to dominant interference ratios where our receiver outperforms the traditional MMSE receiver.

This paper is organized as follows. The system model, interference alignment with combined receivers for heterogeneous 
networks is described in Section II. Section III presents simulation results including a comparison with an MMSE receiver. Conclusions are given in Section IV.

\section{A. Notation}

Throughout this paper, A denotes a matrix, a denotes a vector. $(\cdot)^{*}$ denotes the conjugate transpose. $N(\cdot)$ denotes the null space of a matrix. I denotes an identity matrix.

\section{SYSTEM MODEL}

The proposed IA scheme with combined receivers is described below.

\section{A. Interference Alignment}

The concept of IA for heterogenous cellular uplink systems is given in Fig. 1 and is similar to that described in [8].

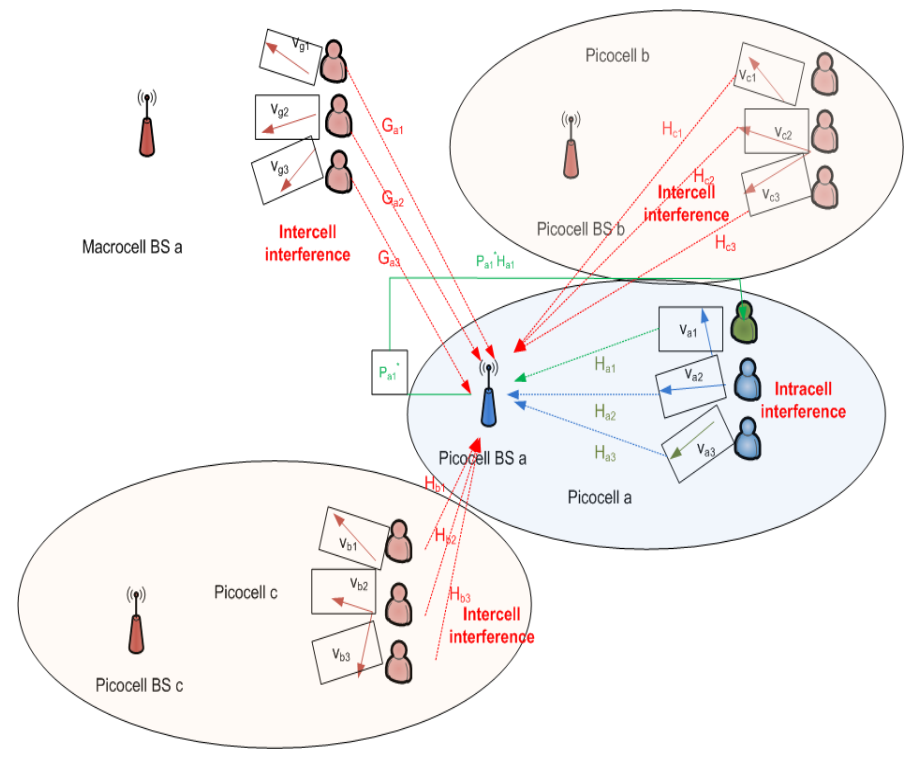

Fig. 1. System Model

Consider the BS of Picocell $a$ where interference will be aligned and mitigated. The received signal at this BS can be written as follows:

$$
\begin{aligned}
y_{a} & =\sum_{i=1}^{K}\left(\sqrt{\rho_{a, i}} \mathbf{H}_{a, i} \mathbf{v}_{a, i} x_{a, i}+\sqrt{\rho_{b, i}} \mathbf{H}_{b, i} \mathbf{v}_{b, i} x_{b, i}\right. \\
& \left.+\sqrt{\rho_{c, i}} \mathbf{H}_{c, i} \mathbf{v}_{c, i} x_{c, i}+\sqrt{\rho_{g, i}} \mathbf{G}_{a, i} \mathbf{v}_{g, i} x_{g, i}\right)+\mathbf{N}_{a},
\end{aligned}
$$

where $K$ is the number of users in each cell, $\mathbf{N}_{a}$ is additive white Gaussian noise (AWGN) which is normalized to variance 1 and $\rho_{a, i}, \rho_{b, i}, \rho_{c, i}$ and $\rho_{g, i}$ are the mean powers of the channels $\mathbf{H}_{a, i}, \mathbf{H}_{b, i}, \mathbf{H}_{c, i}, \mathbf{G}_{a, i}$ respectively, where

- $\mathbf{H}_{a, i}$ : The channel of the $i$ th user in Picocell a to its own BS.

- $\mathbf{H}_{b, i}, \mathbf{H}_{c, i}, \mathbf{G}_{a, i}$ : The channels of the $i$ th interfering user in picocell $b, c$ and macrocell to the BS of picocell $a$ respectively.
Ideally, channels are assumed to be perfectly known at both transmitters and receivers. However, applying some channel estimation errors will naturally result in performance degradation. The users transmit their precoded data, $x$, using the vectors $\mathbf{v}$, as shown in Fig 1, where the subscripts denote the originating cell. The BS uses a postcoding vector $\mathbf{P}_{a, l}$ to decode the lth user's signal as follows:

$$
\begin{aligned}
\tilde{x}_{a, l} & =\mathbf{P}_{a, l}^{*} y_{a} \\
& =\mathbf{P}_{a, l}^{*}(\underbrace{\sqrt{\rho_{a, l}} \mathbf{H}_{a, l} \mathbf{v}_{a, l} x_{a, l}}_{\text {Desired Signal }}+\underbrace{\sum_{i=1, i \neq l}^{K}\left(\sqrt{\rho_{a, i}} \mathbf{H}_{a, i} \mathbf{v}_{a, i} x_{a, i}\right)}_{\text {Intracell Interference }} \\
& +\underbrace{\sum_{i=1}^{K}\left(\sqrt{\rho_{b, i}} \mathbf{H}_{b, i} \mathbf{v}_{b, i} x_{b, i}+\sqrt{\rho_{c, i}} \mathbf{H}_{c, i} \mathbf{v}_{c, i} x_{c, i}\right.}_{\text {Intercell Interference }} \\
& +\underbrace{\left.\sqrt{\rho_{g, i}} \mathbf{G}_{a, i} \mathbf{v}_{g, i} x_{g, i}\right)}_{\text {Intercell Interference }}+\underbrace{\mathbf{N}_{a}}_{\text {Noise }}) .
\end{aligned}
$$

For concreteness, we consider 3 picocells and one macrocell with equal number of users. However, this can be generalized to any system dimension as required.

Considering (2), the BS needs to align the interference coming from other users in other cells (intercell interference) as well as the interference caused by the users in the same picocell (intracell interference). The users transmit their signals with precoding vectors so that undesired signals can be aligned at the BS. Thus, the intracell interference is aligned via the precoding vectors, $\mathbf{v}$. Furthermore, the intercell interference is mitigated with postcoding vectors and the combined receiver. This is explained in Section II-C.

\section{B. Scenarios Considered}

We consider 3 scenarios to evaluate the performance of the proposed combined receiver. In all three scenarios, we consider 3 users in each cell. Each user is equipped with $N_{t}$ antennas, while each BS has $N_{r}$ antennas. We assume there are some dominant interferers with mean total power, $\rho_{\text {dom }}$, while the remaining interferers are weaker with mean total power $\rho_{\text {rem }}$. Similar to [8], we parameterize the system using:

$$
\gamma=\frac{\rho_{\mathrm{rem}}}{\rho_{\mathrm{dom}}} .
$$

In Scenario 1, the BS of picocell $a$ nulls out only 1 interfering macrocell user (assumed dominant) via ZF while MMSE processing reduces the effects of interference caused by the remaining 2 macrocell users and other interfering users in picocell $b$ and picocell $c$. The mean power of the channel of the dominant macrocell user (say user 1) is $\rho_{g, 1}=\frac{\text { SNR }}{N_{t}}$ where SNR is the Signal to Noise Ratio. Since we have normalized the noise variance to $1, \mathrm{SNR}$ is equivalent to the power. The mean channel powers of users in picocells $b$ and $c$ are assumed to be equal to each other $\rho_{b, i}=\rho_{c, i}=\psi_{1} \rho_{g, 1}, \quad(i=1, \ldots, K)$. The mean channel power for the remaining two users in the macrocell is given as $\rho_{g, 2}=\psi_{2} \rho_{g, 1}$ and $\rho_{g, 3}=\psi_{3} \rho_{g, 1}$ 
respectively. We assume the coefficients $0<\psi_{1}, \psi_{2}, \psi_{3}<1$ as all these interferers are assumed weaker.

Scenarios $\mathbf{2}$ and $\mathbf{3}$ are the most aggresive and are considered to evaluate the gains of the combined receiver. Here, we assume that the interfering users' powers are much stronger than in Scenario 1. In Scenarios 2 and 3 we assume that all 3 users in macrocell $a$ and picocell $b$ are dominant and the mean powers of their channels are equal to the mean power of the desired user's channel $\left(\rho_{a, i}=\rho_{g, i}=\rho_{b, i}=\frac{\mathrm{SNR}}{N_{t}}\right)$. The interference caused by the users of picocell $c$ is considered as weak remaining interference. All users of this picocell have the same mean power, $\rho_{c, i}=\gamma\left(\rho_{g, i}+\rho_{b, i}\right), \quad(i=1, \ldots, K)$.

In Scenario 2, we assume that the BS uses ZF to null out all of the dominant user interference while applying MMSE to the remaining interference.

In Scenario 3, the BS nulls out the interference from only 2 users in each dominant interfering cell. The rest of the interference is handled by MMSE processing.

As discussed in Section II.C, Scenario 2 requires more antennas at the receiver than Scenario 3.

\section{Combined Receivers}

In this section, we explain the design of the combined ZF/MMSE receiver. Due to the page limits, only Scenarios 2 and 3 are described below as these are the most complex. The simplification of the equations to Scenario 1 is trivial and therefore omitted. In order to reduce the notational complexity, we drop the mean power of the channels in the equations, as all interference levels are equal for both scenarios.

Our aim is to null the dominant interferers from picocell $b$ and the macrocell via $\mathrm{ZF}$ and reduce the remaining interference plus noise with MMSE. We denote the postcoding vector at the BS for the $l$ th user's signal as

$$
\mathbf{P}_{a, l}=\frac{\tilde{\mathbf{U}}_{a, l} \mathbf{W}_{a, l}}{\left\|\tilde{\mathbf{U}}_{a, l} \mathbf{W}_{a, l}\right\|},
$$

where $\tilde{\mathbf{U}}_{a, l}$ and $\mathbf{W}_{a, l}$ are the ZF part and the MMSE part of the receiver respectively.

1) ZF Part of the Receiver: We first take the singular value decomposition of the dominant interference channel matrices for picocell $a$. This gives

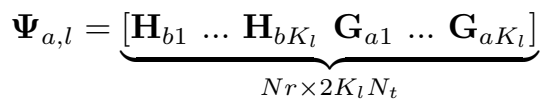

$$
\begin{aligned}
& =\underbrace{\left[\mathbf{U}_{a, l}\right]}_{N_{r} \times N_{r}} \underbrace{\left[\mathbf{D}_{a, l}\right]}_{N_{r} \times 2 K_{l} N_{t}} \underbrace{\left[\mathbf{V}_{a, l}^{*}\right]}_{2 K_{l} N_{t} \times 2 K_{l} N_{t}},
\end{aligned}
$$

where * denotes the conjugate transpose of the matrix. We assume that $N_{t}$ and $N_{r}$ are the same for all sources and receivers for simplicity. $K_{l}$ is the number of dominant interfering users from each cell to be nulled out with ZF. In (5) the matrix,
$\mathbf{D}_{a, l}$, is defined as

$$
\mathbf{D}_{a, l}=\left[\begin{array}{ccc}
\sqrt{\lambda_{1}} & 0 & \cdots \\
\vdots & \ddots & \vdots \\
0 & \cdots & \sqrt{\lambda_{2 K_{l} N_{t}}} \\
0 & \cdots & 0 \\
\vdots & \ddots & \vdots \\
0 & \cdots & 0
\end{array}\right] .
$$

Here the last $N_{r}-2 K_{l} N_{t}$ rows of $\mathbf{D}_{a, l}$ are zeros. This should not be less than the number of interfering streams to be nulled with ZF which is $2 K_{l} N_{t}$. Therefore $N_{r} \geq 4 K_{l} N_{t}$. Using this, $N_{r} \geq 12 N_{t}$ and $N_{r} \geq 8 N_{t}$ for Scenarios 2 and 3 respectively.

We can denote $\mathbf{U}_{a, l}$ as:

$$
\mathbf{U}_{a, l}=[\underbrace{\overline{\mathbf{U}}_{a, l}}_{N_{r} \times 2 K_{l} N_{t}} \mid \underbrace{\tilde{\mathbf{U}}_{a, l}}_{N_{r} \times\left(N_{r}-2 K_{l} N_{t}\right)}],
$$

where $\tilde{\mathbf{U}}_{a, l}$ is the ZF part of the receiver.

2) MMSE Part of the Receiver: The MMSE Part of the receiver denoted as $\mathbf{W}_{a, l}$ in (4) aims to reduce the remaining interference from the other picocells and also AWGN.

The equivalent desired channels can be written as:

$$
\tilde{\mathbf{H}}_{a, l}=\tilde{\mathbf{U}}_{a, l}^{*} \mathbf{H}_{a, l} \mathbf{v}_{a, l},
$$

where $\mathbf{v}_{a, l}$ is the precoding vector. Similarly, we can write the equivalent interference channels from picocells $b, c$ and the macrocell as

$$
\begin{aligned}
\tilde{\mathbf{H}}_{b, i} & =\tilde{\mathbf{U}}_{a, l}^{*} \mathbf{H}_{b, i} \mathbf{v}_{b, i}, \\
\tilde{\mathbf{H}}_{c, i} & =\tilde{\mathbf{U}}_{a, l}^{*} \mathbf{H}_{c, i} \mathbf{v}_{c, i}, \\
\tilde{\mathbf{G}}_{a, i} & =\tilde{\mathbf{U}}_{a, l}^{*} \mathbf{G}_{a, i} \mathbf{v}_{g, i} .
\end{aligned}
$$

Finally, we have the MMSE stage achieved by

$$
\mathbf{W}_{a, l}=(\underbrace{\tilde{\mathbf{H}}_{a, l} \tilde{\mathbf{H}}_{a, l}^{*}}_{\text {Desired }}+\boldsymbol{\Omega}+\underbrace{\sigma^{2} \mathbf{I}}_{\text {Noise }})^{-1} \tilde{\mathbf{H}}_{a, l},
$$

where $\Omega$ is the interference to be reduced by the MMSE processing, given as

$$
\begin{aligned}
\boldsymbol{\Omega} & =\sum_{i=1}^{K} \tilde{\mathbf{H}}_{c, i} \tilde{\mathbf{H}}_{c, i}^{*}+\sum_{i=K_{l}+1}^{K} \tilde{\mathbf{H}}_{b, i} \tilde{\mathbf{H}}_{b, i}^{*} \\
& +\sum_{i=K_{l}+1}^{K} \tilde{\mathbf{G}}_{a, i} \tilde{\mathbf{G}}_{a, i}^{*} .
\end{aligned}
$$

For Scenario 2, all picocell $b$ and macrocell users are nulled out with the ZF part $\left(K_{l}=K\right)$, (13) can be rewritten for Scenario 2 as $\boldsymbol{\Omega}=\sum_{i=1}^{K} \tilde{\mathbf{H}}_{c, i} \tilde{\mathbf{H}}_{c, i}^{*}$.

\section{Proposed IA Algorithm of the Combined Receiver}

We now describe the proposed IA scheme.

- Initialization: The BS initializes its postcoding vector for the $i$ th user in Picocell $a$ as:

$$
\mathbf{P}_{a, l}^{(0)}=\tilde{\mathbf{U}}_{a, l} \mathbf{W}_{a, l}^{(0)},
$$


and

$$
\begin{aligned}
\mathbf{W}_{a, l}^{(0)} & = \\
& \left(\tilde{\mathbf{H}}_{a, l}^{(0)} \tilde{\mathbf{H}}_{a, l}^{(0) *}+\boldsymbol{\Omega}+\sigma^{2} \mathbf{I}\right)^{-1} \tilde{\mathbf{H}}_{a, l}^{(0)},
\end{aligned}
$$

where

$$
\tilde{\mathbf{H}}_{a, l}^{(0)}=\tilde{\mathbf{U}}_{a, l}^{*} \mathbf{H}_{a, l} \mathbf{v}_{a, l}^{(0)}
$$

In (16), we set $\mathbf{v}_{a, l}^{(0)}$ as the maximum eigenvector of $\mathbf{H}_{a, l}^{*} \mathbf{H}_{a, l}$. The BS then feeds back the equivalent channel $\mathbf{P}_{a, l}^{(0) *} \mathbf{H}_{a, l}$ to the respective user.

- Designing Precoding Vectors: The precoding vectors are generated to align the intercell interference at the BS. These should satisfy the following conditions:

$$
\begin{aligned}
& \tilde{\mathbf{v}}_{a, 1}^{(1)} \subset N\left(\left[\mathbf{P}_{a, 2}^{*} \mathbf{H}_{a, 1} ; \mathbf{P}_{a, 3}^{*} \mathbf{H}_{a, 1}\right]\right), \\
& \tilde{\mathbf{v}}_{a, 2}^{(1)} \subset N\left(\left[\mathbf{P}_{a, 1}^{*} \mathbf{H}_{a, 2} ; \mathbf{P}_{a, 3}^{*} \mathbf{H}_{a, 2}\right]\right), \\
& \tilde{\mathbf{v}}_{a, 3}^{(1)} \subset N\left(\left[\mathbf{P}_{a, 1}^{*} \mathbf{H}_{a, 3} ; \mathbf{P}_{a, 2}^{*} \mathbf{H}_{a, 3}\right]\right),
\end{aligned}
$$

where $N(\mathbf{A})$ denotes an orthonormal bases for the null space of the matrix $\mathbf{A}$. These precoding vectors should be selected from the subset vectors of the null space of which size is dependent on the number of dimensions. Then these precoding vectors are normalized as:

$$
\mathbf{v}_{a, i}^{(1)}=\frac{\tilde{\mathbf{v}}_{a, i}^{(1)}}{\left\|\tilde{\mathbf{v}}_{a, i}^{(1)}\right\|} .
$$

- Iteration: In this step, scheduling may also be applied as in [8] and the users can be selected to maximize the capacity. Then the selected users inform the BS of their precoding vectors $\mathbf{v}_{a, i}^{(1)}$ via precoded pilots. Each BS updates the postcoding vector as follows:

$$
\mathbf{P}_{a, l}^{(1)}=\tilde{\mathbf{U}}_{a, l} \mathbf{W}_{a, l}^{(1)},
$$

where

$$
\begin{aligned}
\mathbf{W}_{a, l}^{(1)} & = \\
& \left(\tilde{\mathbf{H}}_{a, l}^{(1)} \tilde{\mathbf{H}}_{a, l}^{(1) *}+\boldsymbol{\Omega}+\sigma^{2} \mathbf{I}\right)^{-1} \tilde{\mathbf{H}}_{a, l}^{(1)},
\end{aligned}
$$

where

$$
\tilde{\mathbf{H}}_{a, l}^{(1)}=\tilde{\mathbf{U}}_{a, l}^{*} \mathbf{H}_{a, l} \mathbf{v}_{a, l}^{(1)} .
$$

The simplification of IA algorithm to Scenario 1 would consist of using only the dominant macrocell user matrix $\mathbf{G}_{a, 1}$ in (5) and then appropriately modifying (10) to include all the remaining interference. Note that as there are $N_{t}$ interfering streams to be nulled with $\mathrm{ZF}, N_{r}$ is $2 N_{t}$ for Scenario 1.

\section{Performance Analysis:}

The performance of the proposed system is gauged by the following metrics.

The Signal-to-Interference Noise Ratio (SINR) for the $l$ th user in picocell $a$ is defined by

$$
\operatorname{SINR}_{a, l}=\frac{\rho_{a, l}\left\|\mathbf{P}_{a, l}^{*} \mathbf{H}_{a, l} \mathbf{v}_{a, l} \mathbf{v}_{a, l}^{*} \mathbf{H}_{a, l}^{*} \mathbf{P}_{a, l}\right\|}{1+\operatorname{INR}_{a, l}},
$$

where $\mathrm{INR}_{a, l}$ is the interference-to-noise ratio which can be written as follows:

$$
\begin{aligned}
\mathrm{INR}_{a, l} & =\left\|\mathbf{P}_{a, l}^{*}\left(\sum_{i=1, i \neq l}^{K} \rho_{a, i} \mathbf{H}_{a, i} \mathbf{v}_{a, i} \mathbf{v}_{a, i}^{*} \mathbf{H}_{a, i}^{*}\right) \mathbf{P}_{a, l}\right\| \\
& +\left\|\mathbf{P}_{a, l}^{*}\left(\sum_{i=1}^{K} \rho_{b, i} \mathbf{H}_{b, i} \mathbf{v}_{b, i} \mathbf{v}_{b, i}^{*} \mathbf{H}_{b, i}^{*}\right) \mathbf{P}_{a, l}\right\| \\
& +\left\|\mathbf{P}_{a, l}^{*}\left(\sum_{i=1}^{K} \rho_{c, i} \mathbf{H}_{c, i} \mathbf{v}_{c, i} \mathbf{v}_{c, i}^{*} \mathbf{H}_{c, i}^{*}\right) \mathbf{P}_{a, l}\right\| \\
& +\left\|\mathbf{P}_{a, l}^{*}\left(\sum_{i=1}^{K} \rho_{g, i} \mathbf{G}_{a, i} \mathbf{v}_{g, i} \mathbf{v}_{g, i}^{*} \mathbf{G}_{a, i}^{*}\right) \mathbf{P}_{a, l}\right\| .
\end{aligned}
$$

The second metric is the ergodic sum rate for picocell $a$

$$
C_{a}=\sum_{i=1}^{K} C_{a, i},
$$

where

$$
C_{a, i}=\log _{2}\left(1+\operatorname{SINR}_{a, i}\right) .
$$

\section{E. Antenna Correlation}

The combined receiver requires a large number of antenna for Scenarios 2 and 3. If the required number of antennas are deployed in a compact array then it is desirable to investigate the performance of the combined receiver in the presence of antenna correlation that may arise due to close spacing of the antennas. We use the simple exponential correlation model given in [9] as

$$
\mathbf{H}_{a, i}^{c o r r}=\mathbf{R}^{\frac{1}{2}} \mathbf{H}_{a, i},
$$

where $\mathbf{R}$ is the correlation matrix containing elements $R(\nu, \tau)$ defined by

$$
R(\nu, \tau)=\left\{\begin{array}{ll}
\xi^{\tau-\nu} & \text { if } \nu \leq \tau \\
R(\nu, \tau)^{*} & \text { if } \nu>\tau
\end{array},\right.
$$

where $\nu, \tau=1,2, \ldots N_{r}$ and $\xi$ is a value satisfying $0 \leq|\xi| \leq 1$. The cases of $|\xi|=0$ and $|\xi|=0.6$ indicate spatially independent and correlated channels respectively.

\section{Simulation Results}

We evaluate the performance of the proposed IA scheme with combined receivers through simulations. We analyse the performance of the BS in picocell $a$ for all three scenarios described in Section II-B.

In order to evaluate the scenarios, we need to determine the mean powers of the interferers. Once the location of the picocells is fixed, the random user locations within the macrocell and picocell would then in practice determine the appropriate mean power levels for the dominant and the remaining interferers. In this study, we have not considered random user locations and simply fix the values of the mean power levels of the interferers. This is because the purpose of this study is to evaluate and compare the IA scheme and not to produce system level results. Intracell interference is 
"nulled" via the null space precoding in (17-19). The null space precoding involves 3 users in each cell, therefore the minimum number of transmit antennas is $N_{t}=3$. The number of receive antennas, $N_{r}$, is scenario dependent. Note that scheduling is not considered in the simulations.

In Fig. 2, for Scenario 1, we present the ergodic sum rates as given by (26) of the proposed combined receiver IA approach and compare it with the MMSE based IA approach which is simply the approach given in [8] with a traditional MMSE receiver. We assume $\psi_{1}=0.04, \psi_{2}=0.01$ and $\psi_{3}=0.05$. Hence $\gamma=\frac{\left(\psi_{1}+\psi_{2}+\psi_{3}\right) \rho_{g, 1}}{\rho_{g, 1}}=0.1$. As in the previous discussion, $N_{r}=6$, (i.e. $2 N_{t}$ ) for this scenario. All channels are i.i.d complex Gaussian and the noise is AWGN. The results indicate that, even though we completely

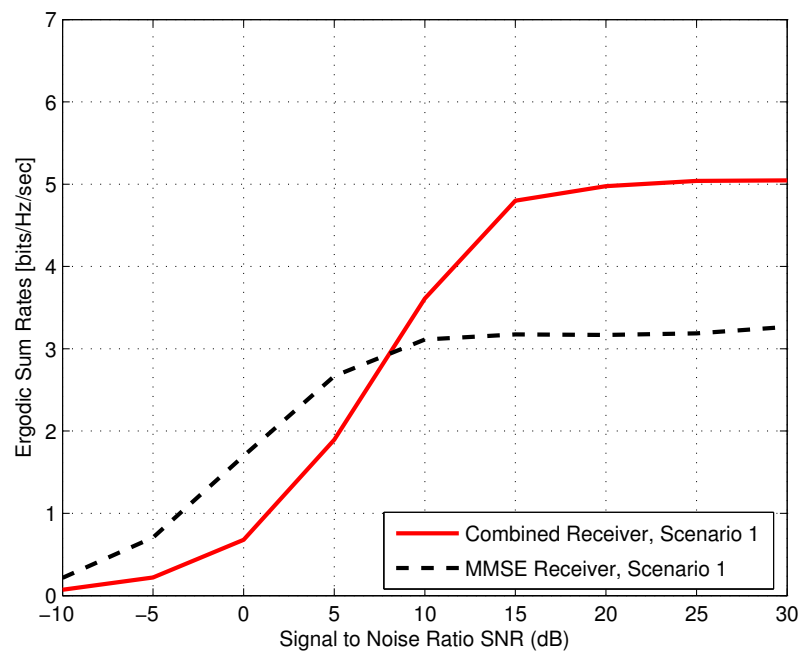

Fig. 2. Ergodic sum rates for scenario $1, N_{t}=3, N_{r}=6$

null out only 1 dominant interferer with the ZF part, the proposed approach achieves better sum rates at higher SNR than the MMSE receiver. For low SNRs there are no dominant interferers, and $\mathrm{ZF}$ is not being utilized to its full potential.

Next, we evaluate the simulations for Scenarios 2 and 3. In Fig. 3, we assume $N_{t}=3$ and $\gamma=0.2$ for both scenarios. Scenarios 2 and 3 have many dominant interferers. Both of these scenarios require a large number of antennas, therefore we consider the impact of antenna correlation. The simulation results in Fig. 3 show that the combined receiver IA significantly outperforms the MMSE receiver IA. The gain in Scenario 3, where we null out fewer dominant interferers, is reduced as compared to Scenario 2. This is intuitively justifiable as some dominant interferers are being added to the remaining interferers and are mitigated via the MMSE part. The correlated results with $|\xi|=0.6$ result in a degradation in the performance. However, we see that the combined receiver still significantly outperforms the MMSE receiver. Simulations not reported in this paper, for higher values of antenna correlation, suggest that the combined receiver continues to maintain its gain over the MMSE receiver. Antenna correlation impacts

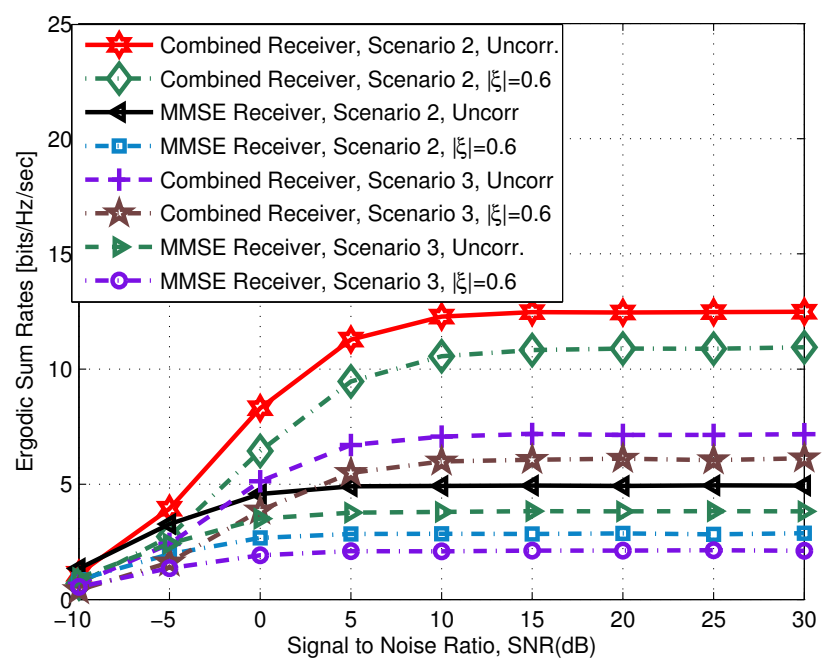

Fig. 3. Ergodic sum rates of both scenario 2 and scenario 3 for $N_{t}=3, \gamma=$ 0.2

the MMSE receiver more significantly than the combined receiver, which is further explained in Fig. 6.

In Fig.4, we investigate which part of the combined receiver is providing the gain. We show the ergodic sum rate for a ZF-only receiver where only the dominant interferers are nulled and the remaining interferers are ignored. We compare this with the combined receiver and the MMSE receiver for Scenario 2. Here we see that it can be beneficial to cancel

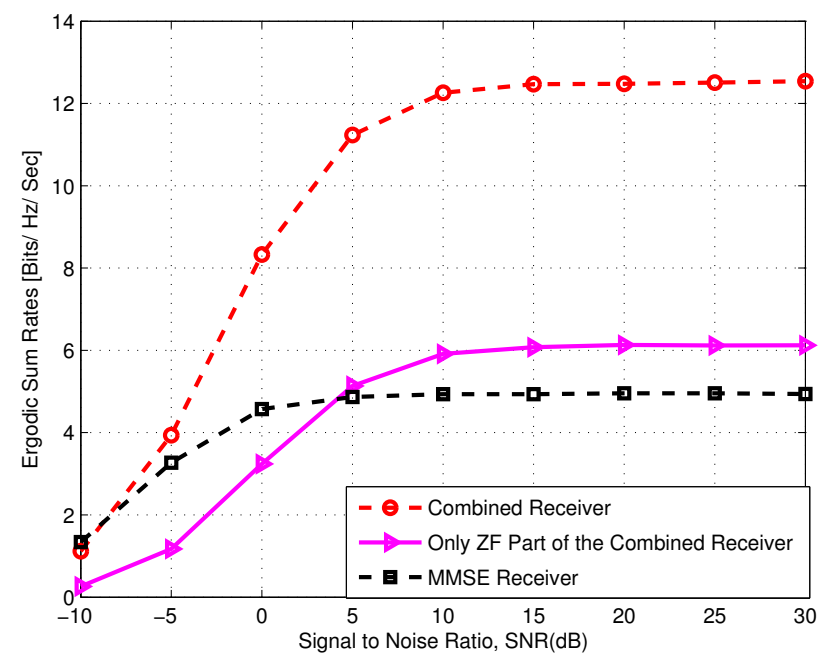

Fig. 4. Ergodic sum rates of scenario 2 using combined receiver, Only ZF part of this combined receiver and MMSE receiver $N_{t}=3, \gamma=0.2$

dominant interferers as the ZF part results in a gain over the MMSE receiver for large SNRs. At lower SNRs, there is no dominant interferer, hence the MMSE receiver is superior. For high SNR, however, when both dominant and remaining interference are present, both receivers are required to obtain 
the large gains.

In Fig. 5, we give the difference between the sum rates of the combined receiver IA and the MMSE IA for different $\gamma$ values for both Scenario 2 and Scenario 3. In doing so, we can analyse the range of $\gamma$ values where the performance of the combined receiver converges to that of the MMSE receiver. We evaluate the simulations for $\mathrm{SNR}=5 \mathrm{~dB}$. The results show that

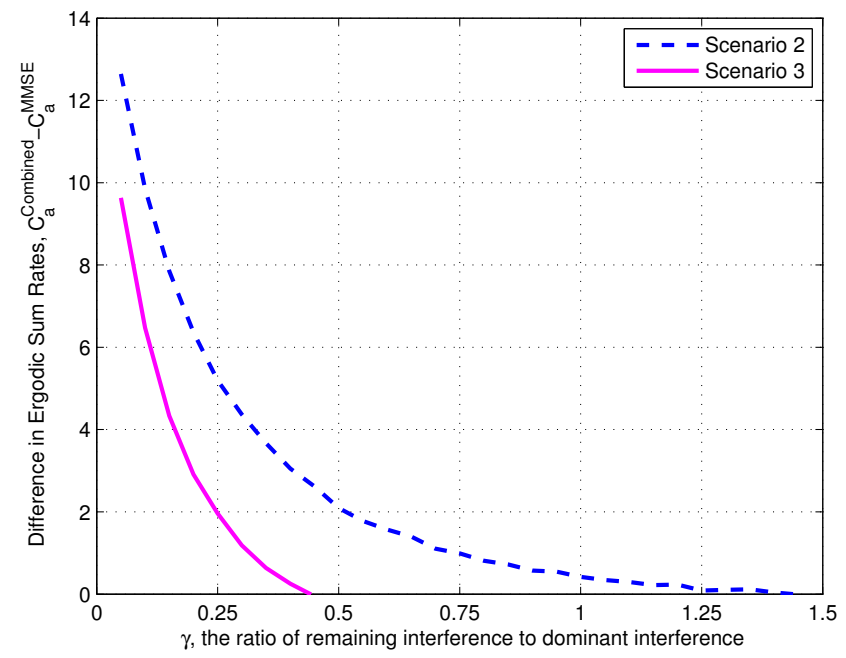

Fig. 5. Difference between the sum rates of proposed combined receivers and MMSE receivers for various $\gamma$ ratios, $\mathrm{SNR}=5 \mathrm{~dB}, N_{t}=3$

the combined receiver IA provides a huge gain when $\gamma$ is close to zero i.e. the remaining interference is very much weaker than the strong dominant nearby interfering cells. However, naturally this gain degrades as $\gamma$ increases. The simulation results also indicate that the combined receiver in Scenario 3 converges to MMSE much quicker than in Scenario 2, as it nulls out less interference with ZF in Scenario 3, relative to Scenario 2.

Finally, we show the cumulative distribution functions (cdfs) of SINR at the BS of picocell $a$ for Scenario 2 in Fig. 6. The results show that the combined receiver achieves better SINR compared to the MMSE receiver IA scheme even with correlated antennas. Furthermore, we observe that the combined receiver is less prone to the effects of antenna correlation, as evidenced by the smaller performance degradation in Fig. 6 .

\section{CONCLUSION}

In this paper, we have proposed a novel receiver which nulls the dominant interfererence via $\mathrm{ZF}$ and reduces the remaining interference and noise through MMSE processing. We have shown that the combined receiver achieves better sum rates and SINR compared to an MMSE receiver. The gain of the combined receiver is impressive but requires a large number of antennas at the BS. Because of this, we have considered antenna correlation and shown with simulation results that the combined receiver is less prone to the effects of antenna correlation.

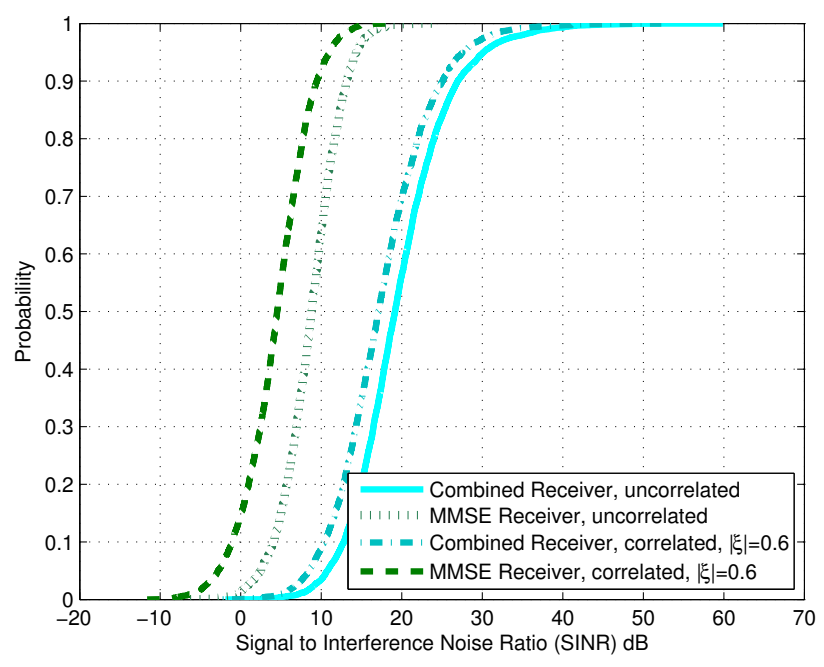

Fig. 6. CDF of signal to interference noise, scenario 2 for correlated and uncorrelated channels, $\gamma=0.2, \quad N_{t}=3$

\section{REFERENCES}

[1] CISCO, "Cisco Visual Networking Index: Global Mobile Data Traffic Forecast Update 2011-2016," FLGD 10229 03/12, Signals and Communication Technology 2012

[2] V. Cadambe and S. Jafar, "Interference Alignment and the degrees of freedom of the $K$ user interference channel," IEEE Trans. on Inf. Theory. Vol. 54, issue 8, pp. 3425-3441, Aug. 2008.

[3] M.A. Maddah Ali, A.S. Motahari and A.K. Khandani, "Communication over MIMO X Channels: Interference Alignment, Decomposition and Performance Analysis," IEEE Trans. on Inf. Theory, vol. 54, issue 8, pp. 3457-3470, Aug. 2008.

[4] S. Jafar and S. Shamai, "Degrees of Freedom Region for the MIMO X Channel," IEEE Trans. on Inf. Theory, vol. 54, issue 1, pp. 151-170, July 2007

[5] T. Gou, S. Jafar, S-W Jeon and S-Y Chung, "Aligned Interference Neutralization and the Degrees of Freedom of the $2 \times 2 \times 2$ Interference Channel," IEEE Trans. on Inf. Theory, vol. 58, issue 7, pp. 4381-4395, July 2012.

[6] N. Lee, W. Shin and B. Clerckx, "Interference Alignment with Limited Feedback on Two-cell Interfering Two-User MIMO-MAC," IEEE Proc. of Int. Symposium of Wireless Communication Systems (ISWCS), pp. 566-570, Aug. 2012

[7] W. Shin, N. Lee, J-B Lim, C. Shin and K. Jang, "Interference Alignment Through User Cooperation for Two-Cell MIMO Interfering Broadcast Channels," IEEE Proc. of Global Communications Conference (GLOBECOM), pp. 120-125, Dec. 2010.

[8] C. Suh, Ho and D. Tse, "Downlink Interference Alignment," IEEE Trans. on Communications, vol. 59, issue 9, pp. 2616-2626, Sept. 2011.

[9] S. Loyka, "Channel Capacity of MIMO Architecture Using the Exponential Correlation Matrix," IEEE Communication Letters, vol.5, pp. 369371, Sept. 2001.

[10] R.F. Ustok, P.A. Dmochowski, P.Smith, M. Shafi, “Aligned Interference Neutralisation for $2 \times 2 \times 2$ Interference Channel with Imperfect Channel State Information," Proc. IEEE Int. Conference on Communications (ICC), pp. 3823-3828, June 2013. 